\title{
QUALIDADE DE GOIABAS 'PALUMA' MINIMAMENTE PROCESSADAS TRATADAS COM 1-METILCICLOPROPENO ${ }^{1}$
}

\author{
ELLEN TOEWS DOLL HOJO², TIYOKO NAIR HOJO REBOUÇAS ${ }^{3}$, ABEL REBOUÇAS SÃO JOSÉ ${ }^{3}$, \\ RONALDO HISSAYUKI HOJO ${ }^{4}$, ANAPAULA PRADO BARRETO 5
}

RESUMO - O objetivo deste trabalho foi determinar os efeitos do 1-MCP na qualidade de goiabas 'Paluma' minimamente processadas, armazenadas a $3^{\circ} \mathrm{C}$. O delineamento estatístico utilizado foi o inteiramente casualizado, em esquema fatorial 3 x 5 , com 3 repetições, em que o primeiro fator correspondeu aos tratamentos: testemunha, aplicação de $1.000 \mathrm{~nL} . \mathrm{L}^{-1}$ de 1 -MCP sobre o fruto intacto, antes do corte, e aplicação de 1.000nL.. $\mathrm{L}^{-1}$ de 1-MCP no produto processado. O segundo fator foi o tempo de armazenamento $(0 ; 3 ; 6 ; 9 \mathrm{e} 12$ dias $)$ a $3^{\circ} \mathrm{C}$. Cada parcela foi composta por 10 metades. O 1-metilciclopropeno (1-MCP) não afetou a qualidade de goiabas 'Paluma' minimamente processadas e armazenadas a $3^{\circ} \mathrm{C}$, durante 12 dias, na concentração e no período de exposição estudados.

Termos para Indexação: Psidium guajava, 1-metilciclopropeno, processamento mínimo.

\section{QUALITY OF 'PALUMA' GUAVAS MINIMALLY PROCESSED TREATED WITH 1-METHYLCYCLOPROPENE}

ABSTRACT - The objective of this work was to determine the effects of 1-MCP in the quality of guava 'Paluma' minimally processed, stored at $3^{\circ} \mathrm{C}$. The experiment was carried out in a completely randomly design, in a $3 \times 5$ factorial scheme, with 3 replicates, in which the first factor corresponded to the treatments: witness, application of 1000nL.L-1 of 1-MCP on the intact fruit, before cutting and application of $1000 \mathrm{~nL} . \mathrm{L}-1$ of $1-\mathrm{MCP}$ in the processed product. The second factor was the period of storage $(0,3,6,9$ and 12 days) at $3^{\circ} \mathrm{C}$. The experimental units were built up with 10 halves of fruits. The 1-MCP did not affect the quality of 'Paluma' guava minimally processed and stored at $3^{\circ} \mathrm{C}$ during 12 days, in the studied concentration and period of exposition.

Index Terms: Psidium guajava, 1-methylcyclopropene, minimum process.

\section{INTRODUÇÃO}

Uma técnica que vem sendo amplamente utilizada no melhor aproveitamento de frutas e hortaliças é o uso do processamento mínimo segundo Chitarra (1998). Esta técnica é uma alternativa utilizada para redução das perdas e melhor utilização da colheita. As perspectivas são promissoras para estes produtos que têm como público-alvo os serviços de fornecimento de alimentos prontos e de preparo rápido.

Entretanto, o processamento mínimo dos frutos produz danos físicos ao mesmo, resultando em respostas físicas e fisiológicas pelo tecido cortado, que podem manifestar-se imediatamente depois do processamento ou durante o armazenamento (Alves et al., 2005). A produção de etileno é estimulada por ações físicas, como descascamento e fatiamento, e as concentrações acumuladas são suficientes para afetar sua qualidade.

O 1-metilciclopropeno (1-MCP), através de seu papel inibitório da ação do etileno, tem- se mostrado efetivo na extensão da vida pós-corte de frutas minimamente processadas, como banana, kiwi, manga e caqui (Vilas Boas \& Kader, 2001). Ele tende a diminuir a taxa respiratória e, principalmente, prevenir o amaciamento desses frutos após o processamento mínimo.

A concentração de 1-MCP necessária para se obter efeito na inibição da ação do etileno varia conforme a espécie, a cultivar, o estádio de maturação, a temperatura de exposição, a interação entre concentração e tempo de exposição e a produção de novos receptores de etileno (Blankenship \& Dole, 2003).

O objetivo deste trabalho foi determinar os efeitos do 1MCP na qualidade de goiabas 'Paluma' minimamente processadas, armazenadas a $3^{\circ} \mathrm{C}$.

'(Trabalho 111-07). Recebido em: 23-04-2007. Aceito para publicação em: 17-03-2008. Fundação de Amparo à Pesquisa do Estado da Bahia FAPESB (BOL0052/2006).

${ }^{2}$ Eng $^{\circ}$. Agr ${ }^{\circ}$., M.Sc. em Ciência dos Alimentos. Bolsista de DTR 2/FAPESB. Universidade Estadual do Sudoeste da Bahia. Estrada do Bem Querer km 04, CEP 45083-900 Vitória da Conquista-BA, Brazil. E-mail: ellendollhojo@yahoo.com.br.

${ }^{3}$ Eng $^{\circ}$. Agr ${ }^{\circ}$., D.Sc., Prof. do Departamento de Fitotecnia e Zootecnia, Universidade Estadual do Sudoeste da Bahia. E-mail: tiyoko@uesb.br; abeljose777@hotmail.com.

${ }^{4}$ Eng $^{\circ}$. Agr ${ }^{\circ}$., M.Sc. em Fitotecnia. Bolsista de DTR2/FAPESB. Universidade Estadual do Sudoeste da Bahia. E-mail: ronaldo.hojo@yahoo.com.br.

${ }^{5}$ Eng $^{\circ}$. Agr ${ }^{\circ}$., Mestranda da Universidade Estadual do Sudoeste da Bahia. E-mail: agroprado@yahoo.com.br 


\section{MATERIAL E MÉTODOS}

Foram utilizados frutos de goiabeiras cv. Paluma, provenientes de uma única área de produção situado em TanhaçuBA. Os frutos foram colhidos no estádio de maturação "de vez" (coloração verde-mate), no período da manhã, cuidadosamente acondicionados em caixas de papelão de $6 \mathrm{~kg}$ e transportados para o Laboratório de Biotecnologia do Departamento de Fitotecnia e Zootecnia, da Universidade Estadual do Sudoeste da Bahia-UESB, distante $140 \mathrm{~km}$ do local da colheita.

Os frutos, com média de $180,79 \mathrm{~g}$, foram selecionados em função do tamanho, cor e ausência de injúrias, e mantidos em ambiente a $20-22^{\circ} \mathrm{C}$ por $1 \mathrm{dia}$, com a finalidade de proporcionar a evolução da coloração interna e o amaciamento da superfície. Após esse período, foram lavados com detergente neutro e sanificados com hipoclorito de sódio a $150 \mathrm{mg}$.. $\mathrm{L}^{-1}$ por 5 minutos, para evitar contaminação durante o corte, em seguida armazenados a $12^{\circ} \mathrm{C}$ por 12 horas, com a finalidade de diminuir o metabolismo. A aplicação do 1-metilciclopropeno (1-MCP) constou da colocação dos frutos em câmara hermética (caixa de poliestireno expandido de 70L) e exposição ao produto Smartfresh ${ }^{\circledR}(0,14 \%$ de i.a.), por 6 horas a $12^{\circ} \mathrm{C}$. Frutos sem 1-MCP também foram mantidos em câmara hermética, por 6 horas a $12^{\circ} \mathrm{C}$, porém sem a aplicação do regulador vegetal.

Os frutos foram processados cortando-os longitudinalmente ao meio e retirando-se suas extremidades, permanecendo o miolo (polpa + sementes) e o epicarpo (casca), em câmara fria a $12^{\circ} \mathrm{C}$. As metades foram enxaguados com água clorada a $20 \mathrm{mg} . \mathrm{L}^{-1}$ e colocados em peneiras plásticas para drenagem por 3 minutos. Terminada essa etapa, foram acondicionadas em bandejas rígidas de poliestireno expandido $(29 \mathrm{~cm} \times 20 \mathrm{~cm})$, envolvidas com o filme de policloreto de vinila (PVC) esticável, auto-aderente e espessura de $10 \mu \mathrm{m}$, e em seguida armazenadas em câmara fria $\mathrm{a} 3{ }^{\circ} \mathrm{C} \pm 1{ }^{\circ} \mathrm{C}$ e $80-90 \%$ de UR, por 12 dias.

O delineamento estatístico foi o inteiramente casualizado (DIC), disposto em esquema fatorial $3 \times 5$, com 3 repetições, onde o primeiro fator correspondeu aos tratamentos: testemunha, a aplicação de $1.000 \mathrm{~nL} \cdot \mathrm{L}^{-1}$ de 1 -MCP sobre o fruto intacto e antes do corte-AC; e aplicação de $1.000 \mathrm{~nL} . \mathrm{L}^{-1}$ de 1 MCP no produto processado. O segundo fator foi o tempo de armazenamento $(0 ; 3 ; 6 ; 9$ e 12 dias). Cada parcela foi composta por 10 metades. Os produtos armazenados foram analisados quanto à Firmeza $(\mathbf{N})$, determinada com o auxílio de penetrômetro TR, modelo WA68, Italy, com ponteira de $8 \mathrm{~mm}$ de diâmetro. Foram feitas quatro medições por metade, na região equatorial, após remoção de pequena porção da casca; Perda de massa (\%), em que se considerou a diferença entre a massa inicial das metades e a obtida a cada intervalo de tempo da amostragem, utilizando-se de balança semi-analítica AND, modelo EK-1200G, Japan, com precisão de 0,1 grama; Teor de Sólidos solúveis ( ${ }^{\circ}$ Brix), determinado por refratometria, em refratômetro manual ATTO Instruments, WYT-4, Hong Kong, segundo a AOAC (1992); Acidez titulável, expressa em g de ac. cítrico. $100 \mathrm{~g}^{-1}$ de polpa, e determinada por titulação com solução de $\mathrm{NaOH}$ a $0,1 \mathrm{M}$, tendo como indicador a fenolftaleína (Instituto Adolfo Lutz, 1985); pH, que foi determinado utilizando-se de um potenciômetro Marte, modelo MB-10 (AOAC, 1992); Relação sólidos solúveis/acidez titulável, obtida pela divisão do teor de sólidos solúveis pela acidez titulável; e Avaliação sensorial (sabor), através de degustação no $12^{\circ}$ dia de armazenamento, por 100 provadores não-treinados, utilizando-se de uma escala de notas. O método sensorial foi o teste de comparação múltipla ou teste de diferença da testemunha (Dutcosky, 1996). Cada provador recebeu uma amostra-padrão (tratamento-testemunha), especificada com a letra P, e 3 amostras codificadas (tratamentos: testemunha, 1MCP aplicado AC; e 1-MCP aplicado DC) que foram comparadas com o padrão, avaliando-se o grau de diferença entre a amostra codificada e o padrão. A descrição das notas foram: $1=$ extremamente melhor que o padrão; 2 = muito melhor que o padrão; 3 = regularmente melhor que o padrão; 4 = ligeiramente melhor que o padrão; $5=$ nenhuma diferença do padrão; $6=$ ligeiramente pior que o padrão; $7=$ regularmente pior que o padrão; $8=$ muito pior que o padrão; $9=$ extremamente pior que o padrão.

Os resultados foram submetidos à análise de variância, pelo teste F. Para a descrição das variáveis em função dos períodos de armazenamento, foram feitas análises de regressão, e os modelos polinominais foram selecionados observando-se a significância do teste $\mathrm{F}$ para cada modelo e seus respectivos coeficientes de determinação.

\section{RESULTADOS E DISCUSSÃO}

A perda de massa foi influenciada apenas pelo período de armazenamento e não foi verificada interação entre os fatores estudados. Foi observado incremento linear da perda de massa, independentemente de tratamentos, durante o período de armazenamento, embora a mesma não tenha chegado a $1 \%$ (Figura 1) e não foi suficiente para causar murchamento ou comprometer a aparência externa da goiaba minimamente processada. Tal perda é atribuída a reações metabólicas, como a respiração e a transpiração do produto, que reduzem a quantidade da água presente no tecido vegetal. Essa perda mínima pode ser atribuída ao uso da embalagem, que restringe as trocas gasosas dos frutos minimamente processados com o meio, criando uma atmosfera modificada em seu interior, e as baixas temperaturas $\left(0^{\circ} \mathrm{C}\right.$ a $\left.5^{\circ} \mathrm{C}\right)$ de armazenamento, que contribuíram para reduzir a perda de massa das frutas e hortaliças minimamente processadas (Brecht, 1995).

A perda de massa é uma variável importante e está diretamente associada com a qualidade do produto. A perda de massa relaciona-se à perda de água, causa principal da deterioração, resultando não somente em perdas quantitativas, mas também na aparência (murchamento e enrugamento), nas qualidades texturais (amaciamento, perda de frescor e suculência) e na qualidade nutricional (Kader, 1992).

Para a variável firmeza, não houve efeito significativo entre os tratamentos utilizados nem do período de armazenamento na goiaba minimamente processada, sendo o valor médio encontrado 87,80N (Tabela 1).

Vários estudos têm verificado que o 1-MCP retarda o 
amaciamento da polpa em frutos in natura (Lima et al., 2006; Corrent et al., 2005; Bassetto et al., 2005; Kim et al., 2001) como também em frutos minimamente processados (Vilas Boas \& Kader, 2001; Alves et al., 2005). Segundo Blankenship \& Dole (2003), diferentes fatores podem influenciar nos tratamentos que utilizam o 1-MCP em produtos hortícolas, principalmente a concentração do 1-MCP necessária para saturar os receptores e competir com o etileno; o tempo de aplicação do tratamento para que o 1-MCP penetre nos tecidos vegetais; a temperatura ideal para que $o$ tratamento seja efetivo em determinado espaço de tempo; e o grau de maturação do produto, pois o 1-MCP não é efetivo em maturação avançada. É possível que, no presente estudo, o 1MCP não tenha exercido efeito sobre a firmeza no fruto intacto e no produto processado devido a um ou mais destes fatores citados anteriormente. Mir et al. (2001) relatam que a afinidade dos sítios de ligação do etileno pelo 1-MCP é diminuída em baixas temperaturas.

O tratamento com 1-MCP não promoveu diferenças significativas na acidez titulável das goiabas minimante processadas, no entanto houve significância no tempo de armazenamento (Figura 2). Acidez titulável no inicio do armazenamento foi $0,55 \mathrm{~g}$ de ácido cítrico. $100 \mathrm{~g}$ de polpa ${ }^{-1}$, a qual diminuiu até o $6^{\circ}$ dia, e aumentou em seguida, até o final do armazenamento. A diminuição no teor dos ácidos orgânicos na goiaba minimamente processada até o $6^{\circ}$ dia de armazenamento sugere que estes serviram como substrato para as reações metabólicas, tais como respiração. Por outro lado, o acréscimo na acidez após o $6^{\circ}$ dia pode sugerir a ocorrência de processo fermentativo.

Alves et al. (2005), trabalhando com melões minimamente processados, também observaram que a aplicação de 1-MCP não afetou o teor de SS, AT e pH. Bassetto et al. (2005) também relataram que goiabas 'Pedro Sato' in natura, tratadas com 900nL.L $\mathrm{L}^{-1}$ de 1-MCP durante 3 horas e armazenadas a $10^{\circ} \mathrm{C}$, o 1 $\mathrm{MCP}$ não influenciaram nos teores de SS, AT e na perda de massa. Contudo, é importante destacar que as respostas dessas características à aplicação de 1-MCP são muito variáveis. Dependendo da espécie ou cultivar, podem ser verificados resultados distintos (Blankenship \& Dole, 2003).

Não houve efeito significativo dos fatores estudados para os teores de sólidos solúveis (SS) (Tabela 1), cujo valor médio obtido foi de $5,11^{\circ}$ Brix, valor menor que aqueles observados por Mattiuz et al. (2003) e Pereira et al. (2003) ao trabalharem com goiabas 'Paluma' minimamente processadas, que esteve em torno de $7,55^{\circ} \mathrm{Brix}$ e $7,20^{\circ} \mathrm{Brix}$, respectivamente. $\mathrm{O}$ comportamento dos teores de sólidos solúveis concorda com o observado por Mattiuz et al. (2003), de que se manteve estável ao longo armazenamento de goiabas 'Paluma' minimamente processadas. Entretanto, em outros trabalhos com goiabas 'Paluma' e 'Pedro Sato' in natura, foi verificado um pequeno acréscimo de SS durante o armazenamento (Bassetto et al., 2005; Ribeiro et al., 2005; Jacomino et al., 2003).

Machado et al. (2004) relataram também a não-influência do 1-MCP sobre essa característica em melões minimamente processados durante o armazenamento. Alguns aspectos do amadurecimento de frutas são completamente suprimidos pela aplicação do 1-MCP, contudo outros que não são totalmente controladas pelo etileno,continuam. Por exemplo, o seu efeito sobre a degradação do amido e acúmulo de açúcares. O mesmo comportamento foi verificado, entretanto, em frutos intactos, como maçã (Rupasinghe et al., 2000), tomate (Moretti et al., 2001), mamão (Jacomino et al., 2002) e banana (Golding et al., 1998), inclusive, com goiaba (Bassetto et al., 2005), porém, em abacaxi e graviola, foram observados efeitos transitórios do 1-MCP em inibir o acúmulo dos sólidos solúveis (Selvarajah et al., 2001; Lima et al., 2001).

Houve efeito significativo entre os tratamentos e o tempo de armazenamento nos valores de $\mathrm{pH}$. Nas metades da testemunha, oscilou de 4,00 para 3,00; no tratamento $1.000 \mathrm{~nL} . \mathrm{L}$ ${ }^{1}$ de 1-MCP aplicado antes do corte (AC) de 3,67 a 3,24 e no tratamento $1.000 \mathrm{~nL} . \mathrm{L}^{-1}$ de 1-MCP aplicado depois do corte (DC) foi de 3,69 a 3,00 (Figura 3). Observa-se que as goiabas minimante processadas submetidas aos três tratamentos sofreram redução de $\mathrm{pH}$, ao longo do armazenamento. Os frutos que receberam 1MCP depois do corte tiveram menor teor de $\mathrm{pH}$. Alterações no $\mathrm{pH}$ podem estar ligadas aos efeitos indesejáveis de elevadas concentrações de $\mathrm{CO}_{2}$, as quais inibem a atividade da succinato desidrogenase, resultando na acumulação do ácido succínico, e conseqüente desarranjo das funções fisiológicas normais (Rolle $\&$ Chism, 1987). No entanto, Machado et al. (2004) não observaram nenhuma diferença significativa em melões minimamente processados, tratados com 1-MCP, durante o armazenamento.

Verificou-se efeito significativo do período de armazenamento sobre a relação entre sólidos solúveis e acidez titulável (SS/AT) (Figura 4). Quanto à relação SS/AT, todos os tratamentos apresentaram aumento em seus valores até o $6^{\circ}$ dia de armazenamento $(12,59)$. Após essa data, houve diminuição na relação SS/AT até o final do armazenamento $(8,18)$. A relação SS/ AT foi influenciada pelo comportamento acidez titulável, pois os teores de sólidos solúveis foram relativamente estáveis durante o período de armazenamento. Os valores apresentados neste trabalho estão próximos aos observados por Mattiuz et al. (2003), estudando também goiabas 'Paluma'minimante processadas.

A relação SS/AT é um dos índices mais utilizados para a determinação da maturação e da palatabilidade dos frutos. Chitarra \& Chitarra (2005) relatam que o equilíbrio entre os ácidos orgânicos e açúcares é muito importante na avaliação do sabor dos frutos. Assim, no presente trabalho, observou-se que o 1MCP não afetou a característica avaliada. Cocozza et al. (2004) observaram que não houve influência dos tratamentos com 1MCP em mangas in natura para esta característica.

No entanto, quanto à análise sensorial, de acordo com o resultado, pode-se afirmar que as amostras tratadas com 1-MCP apresentaram diferença significativa do padrão (testemunha) em relação ao sabor (Tabela 2), apresentando-se sabor ligeiramente melhor que o padrão e indicando que os provadores revelaram maior predileção pela goiaba minimamente processada tratada com 1-MCP. 
TABELA 1- Valores médios da firmeza e dos teores de sólidos solúveis em produtos minimamente processados de goiabas 'Paluma' submetidas a tratamento com 1 -MCP e armazenados a $3 \pm 1^{\circ} \mathrm{C}$. UESB Vitória da Conquista-BA, 2006.

\begin{tabular}{lcc}
\hline Tratamentos (A) & $\begin{array}{c}\text { Firmeza } \\
(\mathbf{N})\end{array}$ & $\begin{array}{c}\text { Sólidos solúveis } \\
\left({ }^{*} \mathbf{B r i x}\right)\end{array}$ \\
\hline Testemunha & $84,22 \mathrm{a}$ & $5,33 \mathrm{a}$ \\
1-MCP aplicado AC & $90,14 \mathrm{a}$ & $5,00 \mathrm{a}$ \\
1-MCP aplicado DC & $89,06 \mathrm{a}$ & $5,00 \mathrm{a}$ \\
\hline Tempo de armazenamento (B) & & \\
\hline 0 & 92,85 & 5,00 \\
\hline 3 & 88,28 & 5,00 \\
\hline 6 & 86,32 & 5,00 \\
\hline 9 & 80,92 & 5,00 \\
\hline 12 & 90,64 & 5,55 \\
\hline Interação & & \\
\hline A x B & $\mathrm{NS}$ & $\mathrm{NS}$ \\
\hline Coeficientes de variação (\%) & 14,74 & 14,58 \\
\hline
\end{tabular}

* Médias seguidas de mesma letra não diferem entre si, a $1 \%$ de probabilidade, pelo teste de Tukey. NS, não-significativo.

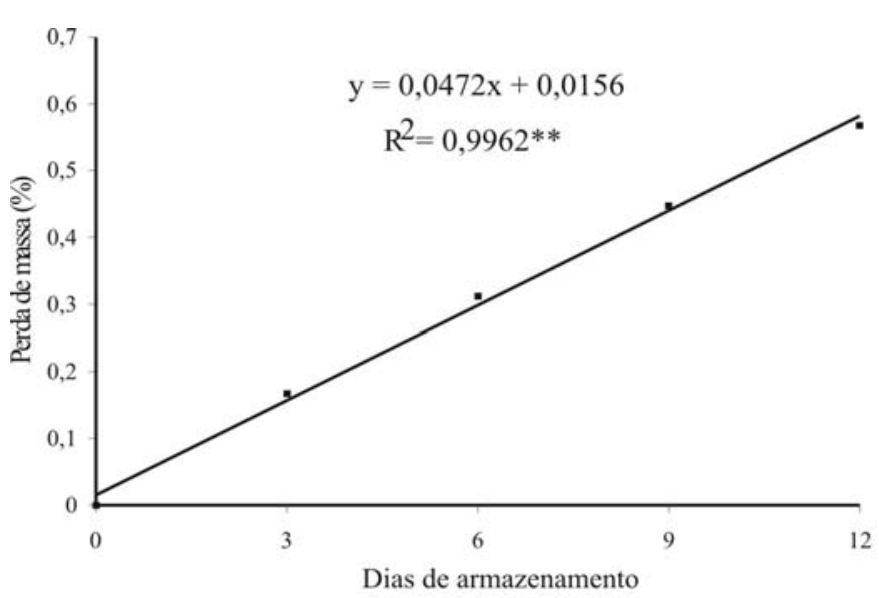

FIGURA 1- Perda de massa em produtos minimamente processados de goiabas 'Paluma' submetidas a tratamento com 1-MCP e armazenados a $3 \pm 1^{\circ} \mathrm{C}$. UESB. Vitória da Conquista-BA, 2006.

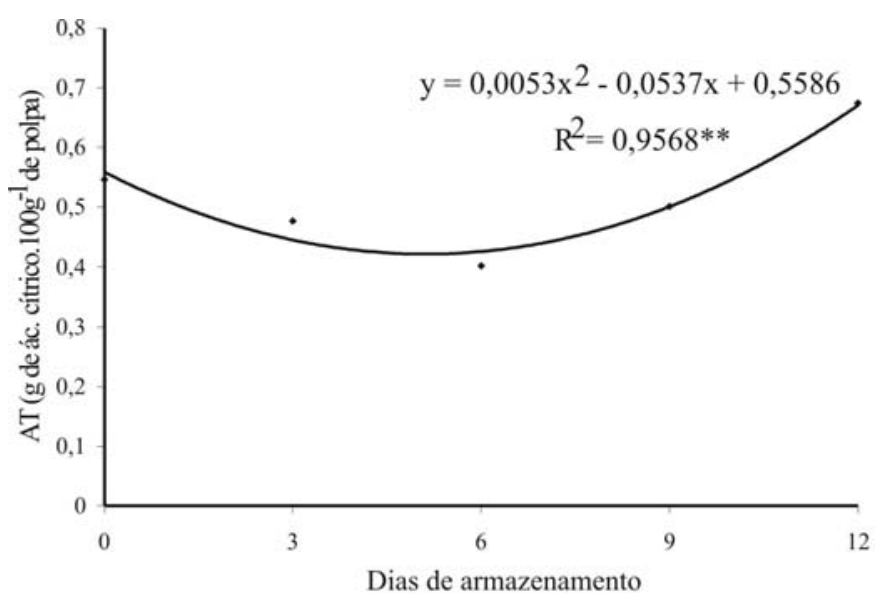

FIGURA 2- Acidez titulável em produtos minimamente processados de goiabas 'Paluma' submetidas a tratamento com 1-MCP e armazenados a $3 \pm 1^{\circ} \mathrm{C}$. UESB, Vitória da Conquista-BA, 2006.
TABELA 2- Análise sensorial em produtos minimamente processados de goiabas 'Paluma' submetidas a tratamento com 1-MCP e armazenados a $3 \pm 1^{\circ} \mathrm{C}$. UESB Vitória da Conquista-BA, 2006.

\begin{tabular}{lc}
\hline Tratamentos $^{*}$ & Sabor \\
\hline Testemunha & $5,54 \mathrm{a}$ \\
1-MCP aplicado AC & $4,61 \mathrm{~b}$ \\
1-MCP aplicado DC & $4,90 \mathrm{~b}$ \\
\hline Coeficientes de variação (\%) & 34,81 \\
\hline
\end{tabular}

* Médias seguidas de mesma letra não diferem entre si, a $5 \%$ de probabilidade, pelo teste de Tukey.

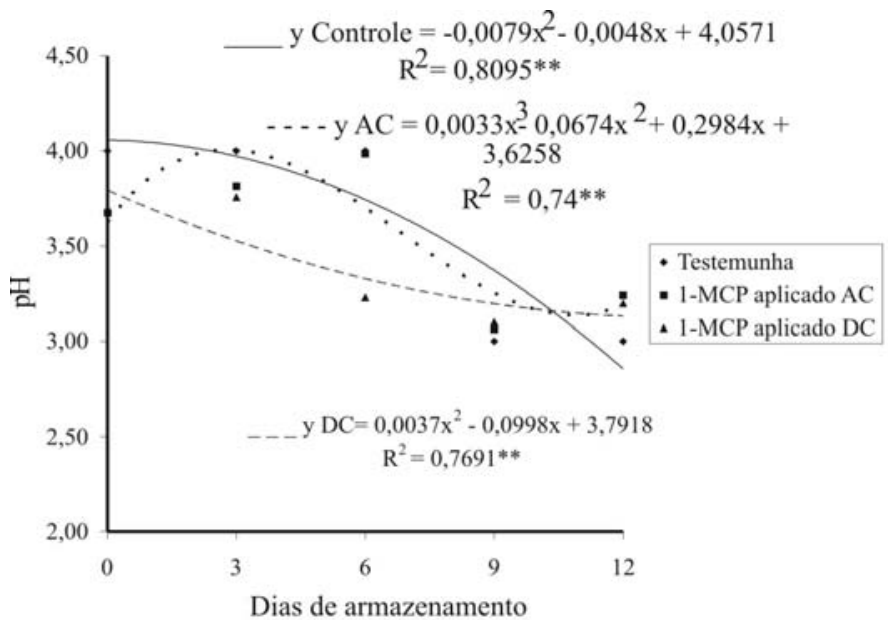

FIGURA 3- $\mathrm{pH}$ em produtos minimamente processados de goiabas 'Paluma' submetidas a tratamento com 1MCP e armazenados a $3 \pm 1^{\circ} \mathrm{C}$. UESB, Vitória da Conquista-BA, 2006.

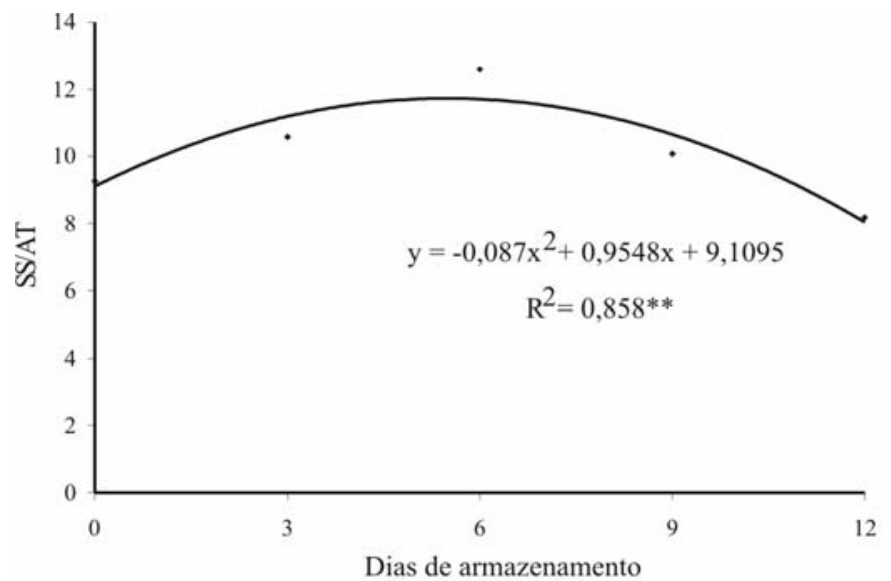

FIGURA 4 -Relação entre sólidos solúveis e acidez titulável (SS AT) em produtos minimamente processados de goiabas 'Paluma' submetidas a tratamento com 1MCP e armazenados a $3 \pm 1^{\circ} \mathrm{C}$. UESB, Vitória da Conquista-BA, 2006. 


\section{CONCLUSÃO}

O 1-metilciclopropeno (1-MCP) não afetou a qualidade de goiabas 'Paluma' minimamente processadas e armazenadas a $3^{\circ} \mathrm{C}$ durante 12 dias, na concentração e no período de exposição estudados.

\section{AGRADECIMENTOS}

À FAPESB, pela concessão de bolsa de DTR 2 e a empresa ROHM IHAAS, pelo fornecimento do 1-MCP.

\section{REFERÊNCIAS}

ALVES, R. E.; VILAS BOAS, E. V. B.; BASTOS, M. S. R.; MACHADO, F. L. C.; SILVA, E. O. Uso de 1-metilciclopropeno (1-MCP) em la conservación de frutas minimamente procesadas. In:GONZÁLEZ-AGUILAR, G. A.; GARDEA, A. A.; CUAMEANAVARRO, F. Nuevas tecnologías de conservación de productos vegetales frescos cortados. Guadarajara: Ciad, 2005. p.415-423.

AOAC - ASSOCIATION OF OFFICIAL ANALYTICAL CHEMISTRY. Official methods of analysis of the Association of Official Analytical Chemistry. $12^{\text {th }}$ ed. Washington, 1992. 1015 p.

BASSETTO, E.; JACOMINO, A. P.; PINHEIRO, A. L. Conservation of 'Pedro Sato' guavas under treatment with 1methylcyclopropene. Pesquisa Agropecuária Brasileira, Brasília, v. 40, n. 5, p. 433-440, Maio. 2005.

BLANKENSHIP, S.; DOLE, J. M. 1-Methylcyclopropene: a review. Postharvest Biology and Technology, Amsterdam, v. 28, p. 1-25, 2003.

BRECHT, J. K. Physiology of lightly processed fruits and vegetables. HortScience, Alexandria, v. 30, n. 1, p.18-22, Feb. 1995.

CHITARRA, M. I. F. Processamento mínimo de frutos e hortaliças. Viçosa: Centro de Produções Técnicas, 1998. 88 p.

CHITARRA, M. I. F.; CHITARRA, A. B. Pós-colheita de frutos e hortaliças: fisiologia e manuseio. 2ed. Ver. e ampl. Lavras: UFLA, $2005.783 \mathrm{p}$.

COCOZZA, F.M.; PEREIRA, M.E.C.;ALVES, R. E.; FILGUEIRAS, H. A. C.; JORGE, J. T. Respiration rate and chemical characteristics of cold stored. Acta Horticulturae, Amsterdam, v. 645, p. 645-650, 2004.

CORRENT, A. R.; PARUSSOLO, A.; GIRARDI, C. L.; ROMBALDI, C. V. Uso do 1-metilcilopropeno no controle da maturação de maçãs cv. 'Royal Gala'. Revista Brasileira de Fruticultura, Jaboticabal, v. 27, n. 2, p.207-210, 2005.
DUTCOSKY, S. D. Análise sensorial de alimentos. Curitiba: Champagnat, 1996. 123p.

GOLDING, J. B.; SHEARER, D.; WYLLIE, S. G.; MCGLASSON, W. B. Application of 1-MCP and propylene to identify ethylenedependent ripening processes in mature banana fruit. Postharvest Biology and Technology, Amsterdam, v. 14, n. 1, p. 87-98, Sept. 1998.

INSTITUTO ADOLFO LUTZ. Normas analíticas: métodos químicos e físico-químicos para análise de alimentos. 2. ed. São Paulo, 1985. v. 1, 371 p.

JACOMINO, A. P.; KLUGE, R. A.; BRACKMANN, A.; CAMARGO E CASTRO, P. R. Controle do amadurecimento e senescência de mamão com 1-metilciclopropeno. Scientia Agrícola, Piracicaba, v. 59, n. 2, p. 303-308, abr.jun. 2002

JACOMINO, A. P.; OJEDA, R. M.; KLUGE, R. A.; SCARPARE FILHO, J. A. S. Conservação de goiabas tratadas com emulsões de cera de carnaúba. Revista Brasileira de Fruticultura, Jaboticabal, v. 25, n. 3, p.401-405, 2003.

KADER, A. A. Postharvest technology of horticultural crops. Davis: University of California, 1992. 296p.

KIM, H. O.; HEWETTI, E. W.; LALLU, N. Softening and ethylene production of kiwifruit reduced with 1-methylcyclopropene. Acta Horticulturae, Amsterdam, v. 553, p. 167-170, 2001.

LIMA, M.A. C.; ALVES, R. E.; FILGUEIRAS, H. A. C.; PEREIRA, M. E. C.; ALMEIDA, A. S.; ENÉAS FILHO, J. Alterações durante a maturação de graviola (Annona muricata L.) submetida a aplicação pós-colheita de 1-MCP. Proceedings of the Interamerican Society for Tropical Horticulture, Orlando, v. 45 , n. 1, p. 1-5, 2001.

LIMA, M. A. C.; SILVA, A. L.; AZEVEDO, S. S. N.; SANTOS, P. de SÁ Tratamentos pós-colheita com 1-metilciclopropeno em manga 'Tommy Atkins': efeito de doses e numero de aplicações. Revista Brasileira de Fruticultura, Jaboticabal, v. 28, n. 1, p.64$68,2006$.

MACHADO, F. L. C.; MAIA, G. A.; FIGUEIREDO, R. W.; ALVES, R. E. Conservação de melão cantaloupe minimamente processado, após aplicação de 1-MCP. Revista Ciência Agronômica, Fortaleza, v. 35, n. 2, p. 394-398, 2004.

MATTIUZ, B.; DURIGAN. J. F.; ROSSI JÚNIOR, O. D. Processamento mínimo em goiabas 'Paluma' e 'Pedro Sato'. 2. Avaliação química, sensorial e microbiológica. Ciência e Tecnologia de Alimentos, Campinas, v. 23, n. 3, p.409-413, 2003.

MIR, N. A.; CURELL, E.; KHAN, N. Harvest maturity, storage temperature and 1-MCP aplication frequency alter firmness retention and chlorophyll fluorescence of 'Redchief 
Delicious'apples. Journal of the American Society for Horticultural Science, Alexandria, v. 126, p.618-624, 2001.

MORETTI, C. L.; ARAUJO, A. L.; MAROUELLI, W. A.; SILVA, W. L. C. Schedulling tomato fruit ripening with 1methylcyclopropene. Proceedings of the Florida State Horticultural Society, Stuart, v. 114, p. 118-121, 2001.

PEREIRA, L. M.; RODRIGUES, A. C. C.; SARANTÓPOULOS, C. I. G. L.; JUNQUEIRA, V. C. A.; CARDELLO, H. M. A. B.; HUBINGER, M. D. Vida-de-prateleira de goiabas minimamente processadas acondicionadas em embalagens sob atmosfera modificada. Ciência e Tecnologia de Alimentos, Campinas, v. 23, n. 3, p.427-433, 2003.

RIBEIRO, V. G.; ASSIS, J. S.; SILVBA, F. F.; SIQUEIRA, P. P. X.; VILARONGA, C. P. P. Armazenamento de goiabas 'Paluma'sob refrigeração e em condição ambiente, com e sem tratamento com cera de carnaúba. Revista Brasileira de Fruticultura, Jaboticabal, v. 27, n. 2, p.203-206, 2005.
ROLLE, R. S.; CHISM, G. W. Physiological consequences of minimally processed fruits and vegetables. Journal of Food Quality, Trumbull, v. 10, p.157-177, 1987.

RUPASINGHE, H. P. V.; MURR, D. P.; PALIYTH, G.; SKOG, L. Inhibitory effect of 1-MCP on ripening and superficial scald development in 'McIntosh' and 'Delicious' apples. Journal of Horticultural Science and Biotechnology, Ashford, v. 75, n. 3, p. 271-276, May 2000.

SELVARAJAH, S.; BAUCHOT, A. D.; JOHN, P. Internal browning in cold-storage pineapples is suppressed by a postharvest application of 1-methycyclopropene. Postharvest Biology and Technology, Amsterdam, v. 23, n. 2, p. 167-170, Nov. 2001.

VILAS BOAS, E. V. de B. ; KADER, A. A. . Effect of 1-MCP on fresh-cut fruits. Perishables Handling Quarterly, California, USA, v. 108, p. 25-25, 2001. 